\title{
Axial segregation in spherical and cylindrical rotating tumblers
}

\author{
Umberto D'Ortona ${ }^{1, \star}$, Nathalie Thomas ${ }^{2}$, and Richard M. Lueptow ${ }^{3,4}$ \\ ${ }^{1}$ Aix-Marseille Université, CNRS, Centrale Marseille, M2P2 UMR 7340, 13451, Marseille, France \\ ${ }^{2}$ Aix-Marseille Université, CNRS, IUSTI UMR 7343, 13451, Marseille, France \\ ${ }^{3}$ Department of Mechanical Engineering, Northwestern University, Evanston, Illinois 60208, USA \\ ${ }^{4}$ Department of Chemical and Biological Engineering, Northwestern University, Evanston, Illinois 60208, USA
}

\begin{abstract}
Monodisperse and bidisperse granular flows are studied in rotating tumblers using DEM. In spherical tumblers, flowing particles' trajectories do not follow straight lines but are curved. At the same time particles near the surface drift toward the pole, inducing two global recirculation cells. Combined with radial segregation, drift and curvature compete to impose the axial segregation pattern: Small-Large-Small (SLS) or Large-SmallLarge (LSL). Fill level, rotation speed and wall roughness influence drift and curvature, and modify the resulting segregation pattern. In cylindrical tumblers, equivalent recirculation cells occur next to the end walls. A second pair of recirculation cells with a weak drift in the opposite direction appears at the center for long enough tumblers. Unlike the sphere case, curvature and drift in the primary cells combine to push large particles toward the end walls, explaining why large particle bands appear at the end walls for axial segregation in cylinder.
\end{abstract}

\section{Introduction}

Mixtures of particles of equal density but different size segregate radially in rotating tumblers in only a few rotations. Subsequently, an axial segregation made of successive bands of small and large particles usually appears. Axial segregation has been studied experimentally and numerically in various geometries (cylinder, sphere, double cone). In cylindrical tumblers, bands of small and large particles emerge with a wavelength of about one tumbler diameter. Large particles always accumulate near the end walls of the tumbler rapidly forming the first bands of segregation. In between them, alternating bands of small and large particles may appear subsequently. In spherical tumblers, the axial segregation is always organised in 3 bands, either in a Small-Large-Small (SLS) or in a Large-SmallLarge (LSL) pattern. The choice of the pattern depends on fill level, particles size ratio, sphere-particles size ratio, rotation speed [1], and the wall roughness of the spherical tumbler [2]. The mechanisms for the axial segregation in spherical and cylindrical tumblers will be addressed in this note.

Discrete Element Method (DEM) simulations are perfomed with a standard linear-spring and viscous damper force model for normal forces. In the tangential direction, the classical Cundall and Strack scheme was used [2, 3], as detailed elsewhere.

Figure 1 shows typical situations where the axial segregation patterns are modified due to particle size $(a+b)$, wall roughness $(a+c, e+f)$, or fill level $(c+d)$ in spherical and cylindrical tumblers.

\footnotetext{
^e-mail: umberto@13m.univ-mrs.fr
}

(a)

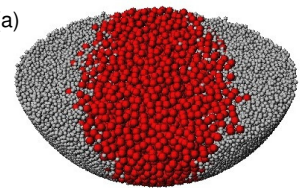

(c)
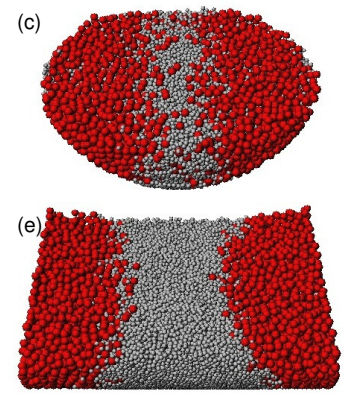

(b)

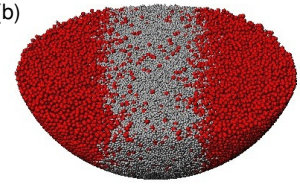

(d)
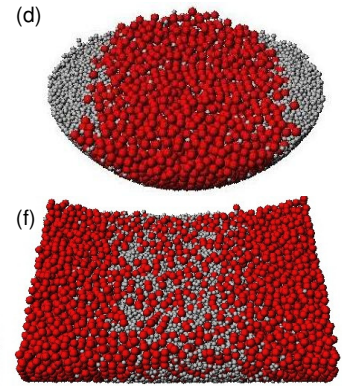

Figure 1. Front view of $14 \mathrm{~cm}$ diameter spherical tumblers or $14 \times 14 \mathrm{~cm}$ cylindrical tumblers filled at $30 \%$ (except $20 \%$ for (d)) with an equal volume bidisperse mixture of particles. All systems rotate at $15 \mathrm{rpm}$ and have reached their stationary segregation pattern. (a) $2+4 \mathrm{~mm}$ particles with smooth walls, the pattern is SLS, (b) 1+2 mm particles with smooth walls leading to LSL, (c) $2+4 \mathrm{~mm}$ particles in a rough tumbler made of $2 \mathrm{~mm}$ glued particles, the pattern is reversed to LSL (d) $2+4 \mathrm{~mm}$ with $2 \mathrm{~mm}$ rough wall tumbler filled at $20 \%$, SLS pattern is obtained, (e) $2+4 \mathrm{~mm}$ particles in a smooth cylindrical tumbler, LSL pattern is obtained, (f) $2+4 \mathrm{~mm}$ particles in a $2 \mathrm{~mm}$ rough cylinder, still LSL but less sharp.

These results have also been obtained experimentally [1]. Fig. 2 shows experiments for conditions equivalent to the simulations (Fig. 1(a, c)) that are obtained in $14 \mathrm{~cm}$ 
diameter spheres with smooth or rough (interior covered by a monolayer of $2 \mathrm{~mm}$ glue particles) walls [2].
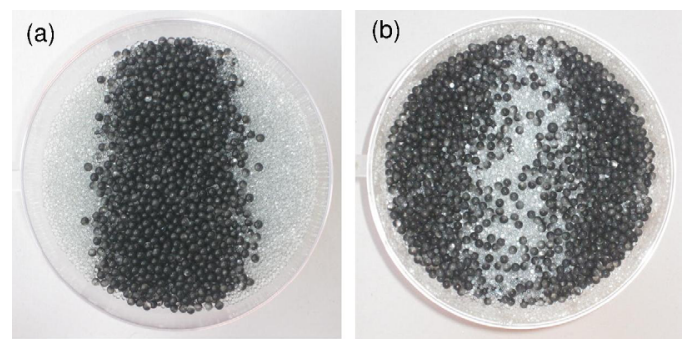

Figure 2. Experimental segregation patterns obtained in a $14 \mathrm{~cm}$ spherical tumblers filled at $30 \%$ with $2+4 \mathrm{~mm}$ particles. Walls are (a) smooth leading to a SLS pattern, or (b) $2 \mathrm{~mm}$ rough leading to LSL (pictures from [2]).

\section{Spherical tumbler}

\subsection{Recirculation cells}

To gain a better understanding on particle trajectories, simulations for monodisperse flow of $2 \mathrm{~mm}$ particles in a $14 \mathrm{~cm}$ spherical drum were performed. Figure 3 shows

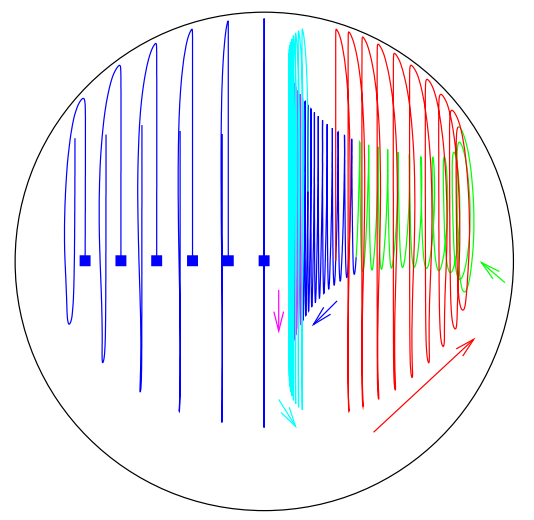

Figure 3. Top view of mean particles trajectories in a $2 \mathrm{~mm}$ rough $14 \mathrm{~cm}$ spherical tumbler. In the left half, trajectories have been integrated for one revolution. In the right half, one trajectory has been integrated for $50 \mathrm{~s}$. Each color corresponds to $10 \mathrm{~s}$. The tumbler is filled at $30 \%$ and rotates at $15 \mathrm{rpm}$. Arrows indicate the direction of drift.

mean trajectories of particles in a spherical rotating tumbler with $2 \mathrm{~mm}$ rough walls. In the left part, 6 trajectories are integrated for one revolution. They start from the static zone, $3 \mathrm{~mm}$ above the bottom wall (squares and vertical straight lines), and enter the flowing layer (curved trajectories), finishing in the static zone again but with a little drift toward the left pole. With this initial position close to the bottom wall, the trajectories flow near the free granular surface. The important facts are: the trajectories in the flowing layer are curved and each trajectory presents a net drift toward the pole.

In the right part of the sphere, one trajectory has been integrated for $50 \mathrm{~s}$. While the particle trajectories are near the surface of the flowing layer (red curve corresponding to $0-10 \mathrm{~s}$ ), the drift is toward the pole. Near the poles the mean trajectory reverses its drift direction flowing near the bottom of the granular layer until it almost reaches the equator (green then dark blue, 10-30s). Then the trajectory reaches the surface of the flow (light blue, 30-50s) and drifts again toward the pole. This trajectory shows that two global recirculation cells are present on a granular flow in a spherical rotating tumbler. Equivalent cells have been obtained in a double cone geometry [4].
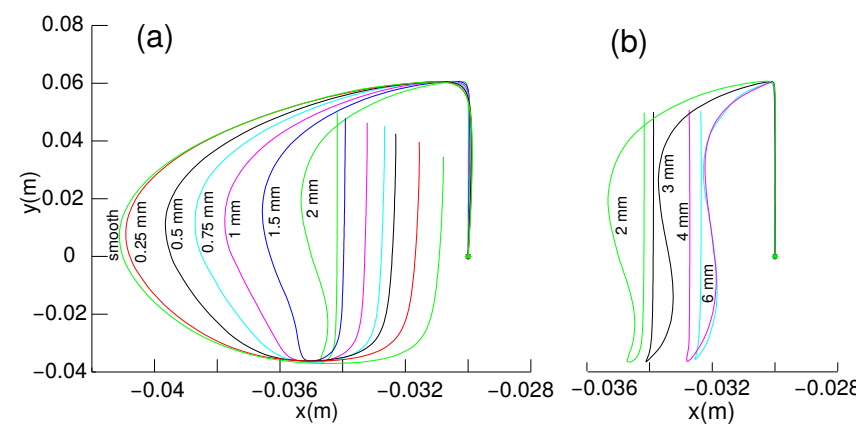

Figure 4. Top view of mean trajectories in $14 \mathrm{~cm}$ tumblers of various roughnesses ranging from (a) completely smooth (labeled 0 ) to a rough wall of $2 \mathrm{~mm}$ glued particles and (b) from $2 \mathrm{~mm}$ to $6 \mathrm{~mm}$ rough walls. The tumbler is filled at $30 \%$ with $2 \mathrm{~mm}$ particles and rotates at $15 \mathrm{rpm}$. Note that the horizontal axes are stretched compared to the vertical one.

Figure 4 compares mean trajectories of particles starting from $x=-0.03 \mathrm{~m}$ with increasing wall roughnesses (from smooth wall to a rough wall of $6 \mathrm{~mm}$ glued particles). The drift, defined as the horizontal distance between the initial position in the static zone and the next pass in that zone, and the curvature, defined as the maximum horizontal distance in one revolution, are modified by the roughness of the wall. With increasing roughnesses, the curvature of the trajectory decreases while the drift increases. For larger roughnesses, the flow trajectories change from simple curves to ones in which the curvature is reversed.

Figure 5 shows particles mean trajectories for two fill levels all along the sphere. For the $20 \%$ full tumbler, trajectories are strongly curved for the smooth wall but have almost no drift. For the rough wall of $2 \mathrm{~mm}$ glued particles, the curvature is less but the drift is larger. Different roughness of the wall induces very different trajectories. For the $50 \%$ full tumbler, the difference in trajectories is smaller.

\subsection{Segregation pattern}

The curvature and drift of particle trajectories explain the origin of the segregation pattern and its inversion from the SLS to the LSL pattern in terms of the competition between two mechanisms. The first mechanism is a consequence of the drift and the recirculation cells. Particles at the surface of the flow drift toward the poles. In a bidisperse granular flow, due to the classical granular radial segregation, the surface is mainly composed of large 

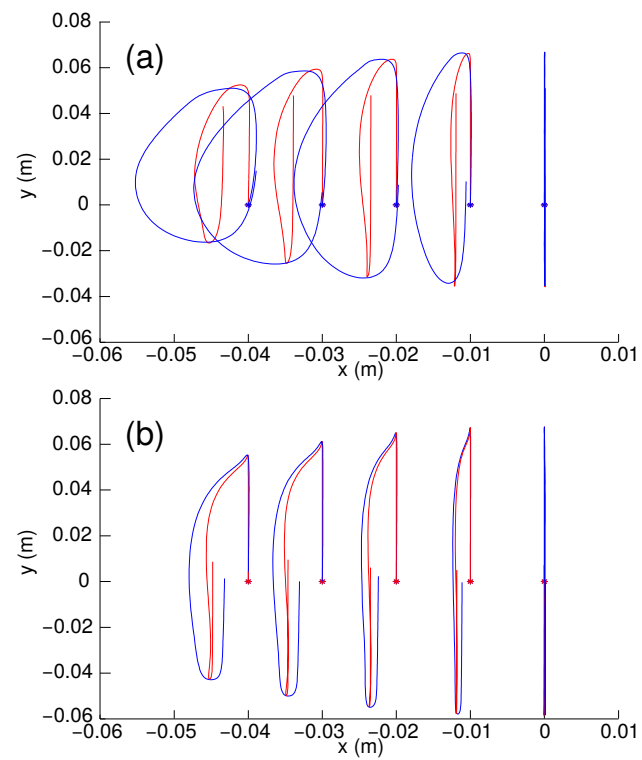

Figure 5. Top view of $2 \mathrm{~mm}$ particles mean trajectories at various positions in a $14 \mathrm{~cm}$ spherical tumblers filled at (a) $20 \%$ and (b) $50 \%$. The spheres have a smooth wall (blue trajectories) or a $2 \mathrm{~mm}$ rough wall (red trajectories) and rotate at $15 \mathrm{rpm}$.

particles. Thus all situations that favor the drift (high fill levels, high roughness) favor the LSL segregation pattern. The second mechanism is linked with the curvature of the trajectories. Fig. 6 shows large (solid lines) and small (dashed lines) particles trajectories starting from the same point $(\times)$ in the flowing zone, flowing along a curved path and finishing their trajectories in the static zone. The side view (a) shows that larger particles remain at the surface due to granular segregation and flow further than small particles that reach the static zone sooner. Consequently, due to the curvature and their longer trajectories, larger particles enter in the static zone closer to the equator of the tumbler, while small particles enter closer to the pole (Fig. 6(b)). Thus the curvature combined with granular segregation favors the SLS segregation pattern. All features that increase the curvature (low fill levels, smooth walls, high rotation speeds) favor the SLS pattern.

\section{Cylindrical tumbler}

The cylindrical tumbler is frequently encountered in industry or in the laboratory. The question that naturally arises is the existence of recirculation cells in cylindrical tumblers.

\subsection{Recirculation cells}

Figure 7 shows monodisperse particle trajectories in the left half of 3 cylindrical tumblers of various lengths. For all cases, recirculation cells (green) develop close to the end walls. If the tumbler is long enough (b), a second pair of recirculation cells (red) develops at the center of the tumbler. For a longer tumbler (c), the secondary convection cells increase in size while the first cells are almost
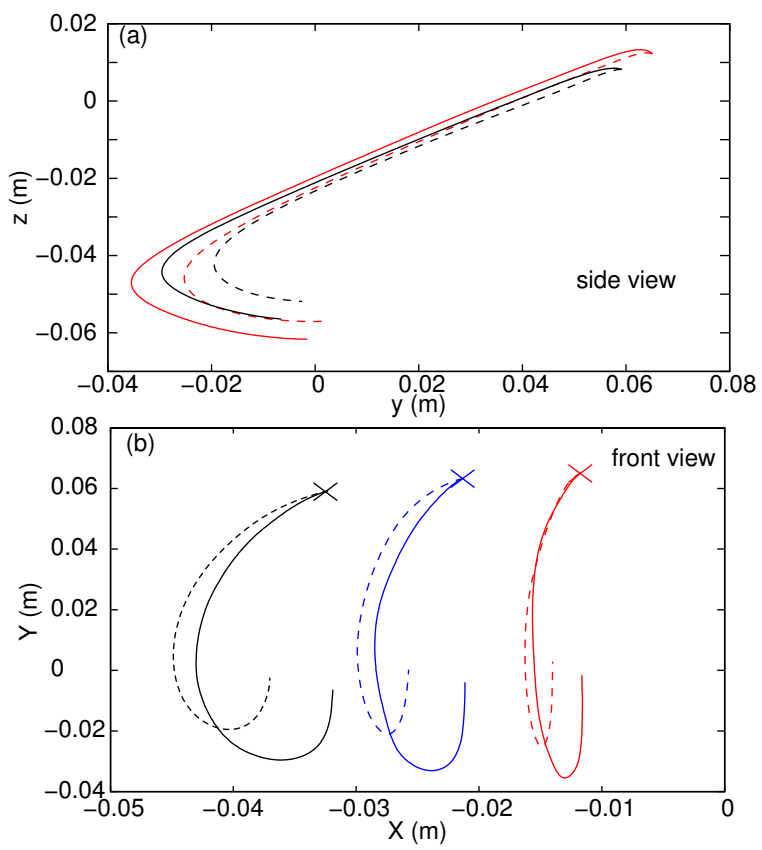

Figure 6. Trajectories of $2 \mathrm{~mm}$ (dashed lines) and $4 \mathrm{~mm}$ (solid lines) particles in a $14 \mathrm{~cm}$ spherical tumblers filled at $30 \%$ and rotating at $15 \mathrm{rpm}$. (a) side view (note that $x-y$ proportions are not the same) and (b) front view. Blue trajectories are omitted in the side view for clarity.
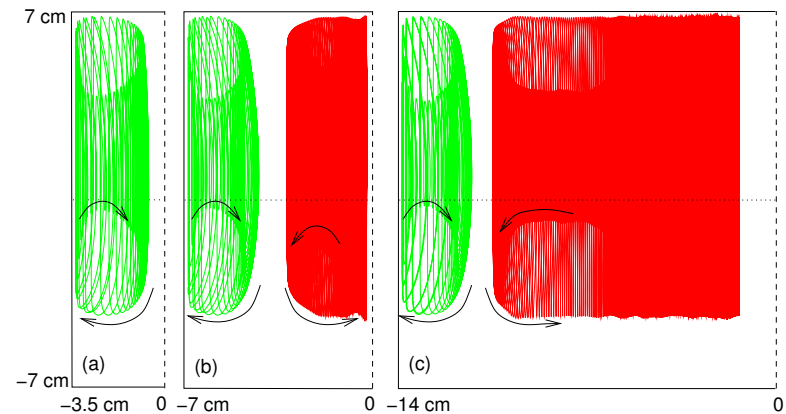

Figure 7. Particle mean trajectories obtained in $14 \mathrm{~cm}$ diameter cylindrical tumblers with lengths of (a) $7 \mathrm{~cm}$, (b) $14 \mathrm{~cm}$ and (c) $21 \mathrm{~cm}$. Tumblers have smooth walls, rotate at $15 \mathrm{rpm}$ and are filled at $30 \%$ with $2 \mathrm{~mm}$ particles. The vertical dashed lines show the center of the tumblers; only the left half of the tumbler is shown, since the trajectories are symmetric. Arrows indicate the direction of recirculation.

unaffected. On the other hand, for short tumblers (a), the secondary center cells disappear. Arrows indicate the direction of circulation in the cell. Primary and secondary cells circulate in opposite directions.

To estimate more accurately the size of each recirculation cell, the drift is measured. Since neighbouring cells circulate in opposite directions, the boundary between two cells corresponds to a null drift. Fig. 8(a) shows the drift for tumblers ranging from 7 to $28 \mathrm{~cm}$ long. The two regions between the cylinder end walls and the intersections with horizontal axis delimit the first cells. Fig. 8(a) confirms that the extent of the cells next to the end walls 

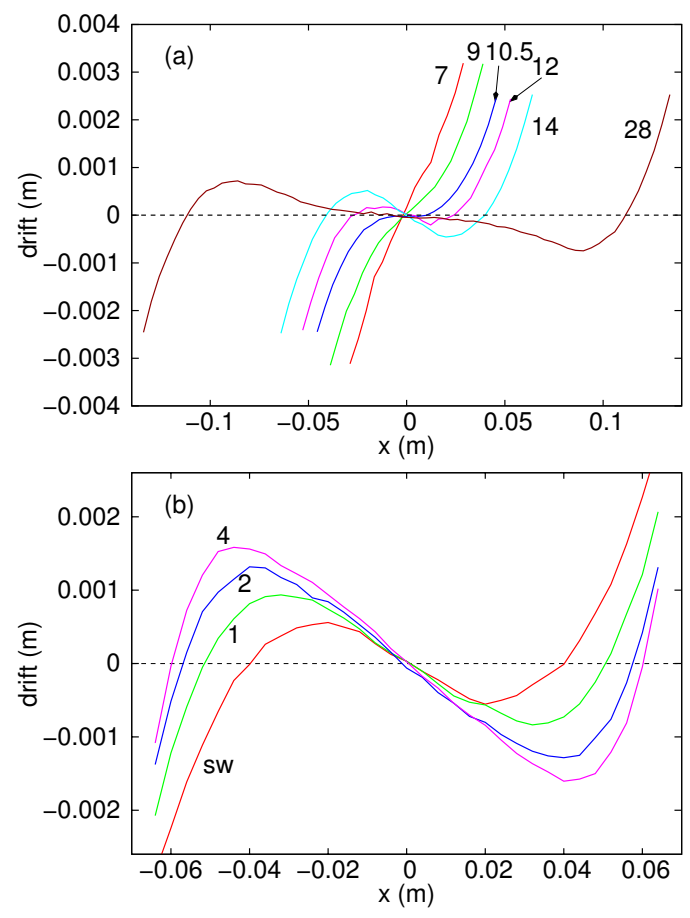

Figure 8. Drift measured along cylindrical tumblers for (a) tumblers of various lengths (7 to $28 \mathrm{~cm}$ ) and (b) $14 \mathrm{~cm}$ cylindrical tumblers made of wall with various roughness (smooth wall (sw) to $4 \mathrm{~mm}$ rough). The boundary between two cells occurs at positions of null drift.

(region where drift $<0$ in the left half) is almost constant, while the secondary cells (region where drift $>0$ ) fill the empty space between the primary cells, and eventually vanish for tumblers of lengths $\leq 10.5 \mathrm{~cm}$. Fig. 8(b) shows the influence of the wall roughness: with increasing roughness, the size of the cell near the endwall decreases.

\subsection{Segregation pattern}

The properties of monodisperse particle trajectories in cylindrical tumblers can be used to explain some features of axial segregation in cylinders. As occurs in a spherical tumbler, the drift pushes particles flowing at the surface toward the poles, which are the end walls here. The trajectory curvatures are inverted compared to the sphere. Thus, the two phenomena are not competing, but both lead to a migration of large particles toward the end wall, explaining why experiments and simulations report the fast emergence of bands of large particles next to the end walls.

The size of the large particle band near the end wall is strongly influenced by the size of the primary cells. This is evident in Fig. 9 showing vertical slices through the bed of particles along a $14 \mathrm{~cm}$ cylinder filled with a mixture of $2+4 \mathrm{~mm}$ particles that undergoes an axial segregation. In the smooth case, the band of large particles next to the end walls captures almost all $4 \mathrm{~mm}$ particles leading to a strong segregation, while for the $4 \mathrm{~mm}$ rough case, the bands of large particles are narrower, and the excess of large particles fills the surface of the flowing layer. The decrease of size of the band with roughness is directly connected to the decrease of the size of the primary convection cells with roughness (Fig. 8(b)).
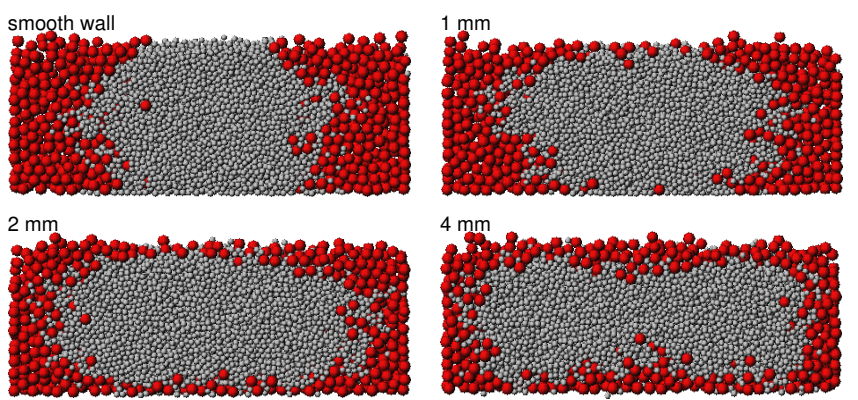

Figure 9. Picture of the segregation pattern $(t=200 \mathrm{~s})$ for horizontal $14 \mathrm{~cm}$ cylinder tumbler filled at $30 \%$ with 2 and $4 \mathrm{~mm}$ particles and rotating at $15 \mathrm{rpm}$. Only particles in a vertical slice including the horizontal axis of rotation are shown. The walls are smooth, or rough made of 1,2 or $4 \mathrm{~mm}$ particles.

\section{Conclusions}

This study of granular flow in spherical and cylindrical tumblers has demonstrated two competing processes responsible for the axial segregation in rotating tumblers: the drift leading to recirculation cells and the curvature of particle trajectories. Wall roughness, rotation speed, and fill level have an influence in both processes. Drift and curvature combined with radial segregation explain the SLS and LSL segregation patterns and the transition between them in a spherical tumbler. In cylindrical tumbler, primary recirculation cells are present next to the end walls, but secondary cells develop only if the cylinder is long enough. In the primary cells, trajectory drift and curvature combine to send large particles toward end walls, explaining why axial segregation in cylinder always has bands of large particles near the end walls, and why the SLS segregation pattern is never obtained in cylinder.

\section{Acknowledgements}

UDO and NT had access to the HPC resource of AixMarseille Université financed by the project Equip@Meso (ANR-10-EQPX-29-01).

\section{References}

[1] P. Chen, B. J. Lochman, J. M. Ottino and R. M. Lueptow, Phys. Rev. Lett. 102, 148001 (2009).

[2] U. D'Ortona, N. Thomas, and R. M. Lueptow, Phys. Rev. E 93022906 (2016).

[3] U. D'Ortona, N. Thomas, Z. Zaman, and R. M. Lueptow, Phys. Rev. E 92062202 (2015).

[4] Z. Zaman, U. D’Ortona, P. B. Umbanhowar, J. M. Ottino, and R. M. Lueptow, Phys. Rev. E 88, 012208 (2013). 